\title{
A stochastic model for filtration by porous materials
}

\author{
Filippo Miele, ${ }^{1,2}$ Pietro de Anna, ${ }^{2, *}$ and Marco Dentz ${ }^{1, \dagger}$ \\ ${ }^{1}$ Spanish National Research Council (IDAEA-CSIC), Barcelona 08034, Spain \\ ${ }^{2}$ Institut des sciences de la Terre, University of Lausanne, Lausanne 1015, Switzerland
}

\begin{abstract}
The transport of colloids in porous media is governed by deposition on solid surfaces and porescale flow variability. Classical approaches, like the colloid filtration theory (CFT), do not capture the behaviors observed experimentally, such as non-exponential steady state deposition profiles and heavy tailed arrival time distributions. In the framework of CFT a key assumption is that the colloid attachment rate $k$ is constant and empirically estimated by a posteriori macroscopic data fitting. We propose a stochastic model that explicitly accounts for variability of the pore-scale transport and attachment properties. Colloidal motion is modeled as a sequence of displacements whose velocity and extension are statistically distributed. Colloidal depositions are modeled as random events whose statistics are determined by flow velocity, pore size and attachment rate. The resulting deposition profiles are in general non-exponential and can be predicted based on the disorder distributions.
\end{abstract}

PACS numbers: 47.56.+r, 92.40.Kf, 05.60.Cd, 05.40.Fb

\footnotetext{
* pietro.deanna@unil.ch

$\dagger$ marco.dentz@csic.es
} 


\section{INTRODUCTION}

The macroscopic phenomenon of filtration is the process by which particles are removed from suspension in fluids: in other words, it is the separation between the suspended and the solid phase of transported colloids [1]. This ubiquitous process takes place in natural (e.g., groundwater and soil [2], hyporheic zone [3]) and industrial systems (e.g. filtration plants [4], pharmaceutical industry and hospital care [5]) and affects many societal issues including health, energy and clear water resources. The removal process of filtration is the composition of a number of small scale mechanisms that include straining, sedimentation, flocculation and surface attachment. In practice, the filtration process requires that a colloid is transported close enough to the host medium's solid surface that it can attach to it $[1]$.

The interaction among suspended colloids, and between colloids and larger solid surfaces is classically described by the DLVO theory [1], which accounts for charged surfaces that interact through a liquid medium. This theory considers the balance between repulsive, Coulomb, and attractive, Van der Waals forces. The former are associated to the so-called double layer that forms around an ionic species in order to maintain electric neutrality and that coats all available surfaces. The latter are due to quantum effects. They quickly vanish with distance and are responsible for the eventual colloid attachment to the grain surfaces [6]. In this framework, when a suspended particle approaches another, or the solid surface of a different object, the ionic clouds overlap and a repulsion force is exerted on the particle preventing them to come closer. However, because of molecular diffusion or hydrodynamics effects, a suspended particle could approach a surface close enough, against the electrostatic repulsion, to feel the attractive force and, eventually, attach to it [1]. These attractive forces take place at very short scales $l$, below the micron (typically below $50 \mathrm{~nm}$ [6]). Thus, they require that the flowing colloids approach the solid boundaries of the grains at distances below $l$ to obtain individual attachment events that lead to the macroscopic filtration phenomenon by the host filter.

Many natural porous media, such as rocks, soil or biological tissues (e.g., wood), as well as industrial porous materials like cements and ceramics, are characterized by their ability to transmit fluids through their pores among solid impermeable grains that constitute the overall medium backbone [7]. These media have been shown to posses a very rich structure in terms of the pore length, pore throat and connectivity( e.g. [8]). This complexity plays a critical role in natural and engineered processes such as groundwater contamination and remediation [9, 10], water infiltration in soils [11] and geologic carbon sequestration [12]. Porous materials represent excellent filters since they are characterized by very large surfaces compared to their volumes [7] and, thus, they are often used as filtration systems [13]. However, pore-scale fluctuations in the medium, flow and deposition properties are represented in the classical Darcy scale colloid filtration theory (CFT) by average quantities like porosity $\phi$, the Darcy velocity $q$ and filtration rate $k[7]$.

Most filtration models rely [14], to the best of our knowledge, on the assumption proposed by Happel that the complexity of flow though a permeable medium can be reduced by considering the porous material as an assemblage of smooth, uniform spheres and representing the whole host medium by set of identical cells each containing a sphere surrounded by a fluid envelope [15]. This concept is schematically represented in Fig 1a. In this framework, each sphere is considered as a collector over which colloids, transported by the flow, may collide when passing close to its solid boundary. In Fig 1a this is represented by the gray area around the black collector. Collisions occur with an attachment or filtration rate $k$ that is associated to the flow and medium properties as $[16,17]: k=3 \eta(1-\phi) q / 2 d_{c}$ where $d_{c}$ is the diameter of the collector (the average grain diameter) and $\eta$ is the collector efficiency (fraction of the flowing colloids that will collide on the collector itself). This rate, $k$, represents the fraction of collision events that result in an attachment event per unit time and is typically measured in units of $[1 / \mathrm{s}]$. Adopting this view of the flow through a porous material, as described by the flow around a set of identical collectors, the efficiency $\eta$ is typically related to the ratio between the cross-sectional area $S_{1}$ of the overall fluid passing around the collector (top gray disk in Fig. 1a) and the surface area $S_{2}$ delimited by the limiting trajectories that will collide due to hydrodynamic and diffusive effects (red disk in Fig. 1a). Different physical models can be considered to define the two surface $S_{1}$ and $S_{2}$ and, thus, $\eta$ (e.g. considering different colloids diffusivity or inertial effects) [18-20]. 
a

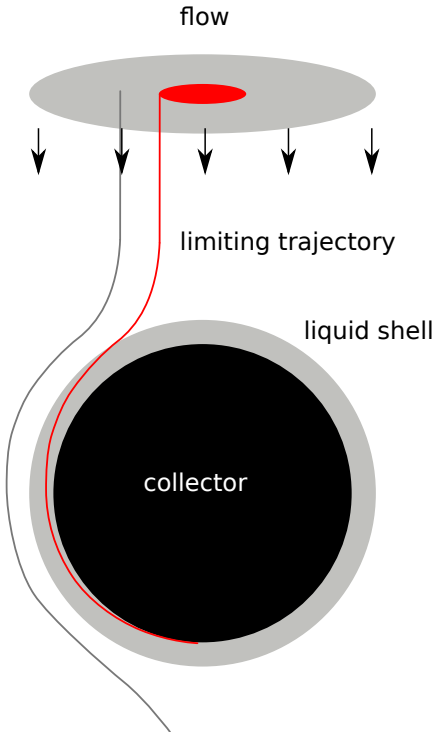

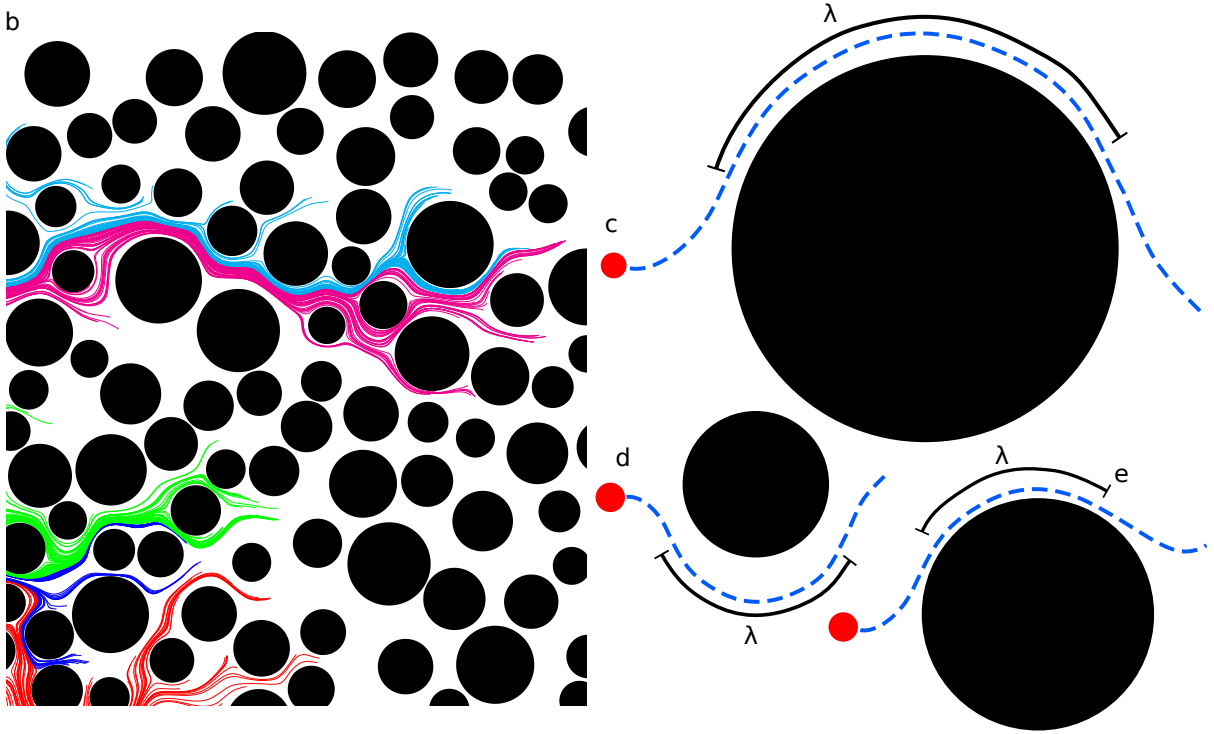

FIG. 1. Filtration models. a. The classical Happel sphere in a cell model based on the porous medium reduction to a single collector. b. Schematic view of our model that takes into account the spatial variability of the collector properties: the porous medium is though as a collection of many collectors (solid grains) around which particles are advected by an heterogeneous velocity field (black disk, adapted from [23]). c-e. Three particles flowing close by solid grain surface: once a transported particle approaches the solid surface it will move along a segment of length $\lambda, \sim$ the size of the grain, keeping a constant velocity $v$, being at a constant distance from the solid surface and, then, experiencing a constant attachment rate $k$.

At the macroscopic scale, filtration is classically studied in terms of breakthrough curves (flux of colloids at fixed location) [21] and deposition profiles (spatial distribution of attached particles at a given time) [22].In the introduced CFT framework, mass conservation can be stated in terms of the classical one-dimensional advection-dispersion equation with the addition of an attachment term modeled through a first-order kinetics, thus, the suspended colloids volumetric concentration $c$ evolves as:

$$
\frac{\partial c}{\partial t}+k c=D \frac{\partial^{2} c}{\partial x^{2}}-v \frac{\partial c}{\partial x},
$$

where $D$ is the hydrodynamic dispersion coefficient and $x$ represent the direction oriented along the mean flow. Neglecting the effect of dispersion, under stationary conditions, Eq. (1) becomes

$$
v \frac{\partial c}{\partial x}=-k c,
$$

whose solution decays exponentially as $c(x) \propto \exp (-k x / v)$. Each filtration event transfers a colloid from the suspended population $c(x)$ to the filtered population, $a(x)$, whose stationary profile is also exponential

$$
a(x) \propto \exp (-k x / v)
$$

The deposition profile dimension is the inverse of a length, $[1 / \mathrm{m}]$ : it can be measured as the number of colloids found in a column slice, of given thickness, at distance $x$ from the inlet. It has been shown that taking into consideration the effect of dispersion, the stationary solutions of (1) also converge to an exponential decay with a slightly different characteristic decay rate [19]. For the sake of simplicity, in the following we will neglect the effects of dispersion.

Filtration experiments showed significant discrepancies between experimental results (mostly from column experiments) and the exponential decay predicted by CFT for the stationary deposition profile (3). In particular, stretched exponential deposition and power law profiles, $a \propto x^{-\alpha}$, have been observed [24, 25]. Some authors proposed to modify the CFT considering that along its own trajectory a transported colloid would experience a given constant attachment rate $k$, but different colloids would experience different $k$ due to chemical heterogeneities [24]. In this 
framework, experimental data are typically fitted by ad-hoc attachment rate distributions without a clear connection to pore-scale properties. Moreover, to properly catch long tails and scale-free behaviors, data should be collected over several orders of magnitude. These type of data, to the best of our knowledge, are not available at the moment. In fact, experiments that investigate the spatial deposition profile $a$ typically are performed in filtration column-beds which, after being flooded with a colloidal suspension for a given period, are dissected into thin slices that are individually analyzed to recover the average number of retained colloids within the considered slice [26]. This methodology, however, is not adequate to measure a deposition profile over several orders of magnitude in the travel distance $x$.

Moreover, while models have been derived [19] to fit some experimental data set through a distribution of attachment rates, they do not account for physical heterogeneity associated to the pore structure and the consequent flow and transport process. Pore structure and flow variability, however, play a central role in the dynamics of pore-scale processes such as particle dispersion and reaction [27-31]. Pore-scale flows are organized in regions of fast and highly variable velocities and zones of stagnation [32-36]. While the high velocities are known to control the overall transmission of fluid, the distribution of low velocities in zones of fluid stagnation have been shown to control and characterize the late-time particle transport [31, 32, 34, 37, 38], mixing and chemical reactivity [39-42].

Here, we propose a model that accounts for the fundamental mechanisms driving filtration in porous media, by explicitly taking into account the variability of colloid transport and attachment rates. We focus on purely advective transport for which the flow field heterogeneity arises from the complex path of streamlines, along which colloids move. As represented schematically in Figure 1b-e, we represent a porous medium as a collection of heterogeneously distributed grains (represented by black disks in Figure 1) around which the trajectories of individual colloids are organized. Thus, we develop a stochastic model that combines the heterogeneous host medium structure, fluid velocity and attachment rate distributions. In particular, as represented in Fig. 1c-e, we assume that in each pore of length $\lambda$ a transported colloid keeps a constant velocity $v$, a constant distance from the grain wall and, thus, a constant attachment rate $k$. Once it moves to the next pore of different size, also $v$ and $k$ will change. This reflects the fact that flow and medium structure may vary across a range of length scales.

\section{STOCHASTIC MODEL}

\section{A. Model formulation}

The basic mechanisms that drive the overall filtration phenomenon are i) the advective displacement of suspended colloids through the pore space, ii) their individual attachment to the grain walls. Purely advective particle motion in the flow field $\mathbf{u}(\mathbf{x})$ is governed by the advection equation

$$
\frac{d \mathbf{x}}{d t}=\mathbf{u}[\mathbf{x}(t)],
$$

where $\mathbf{x}$ represents the position of a transported particle, that can be measured using microscopy and tracking techniques in units of $[\mathrm{m}]$. The traveled distance $s(t)$ from the inlet position of a particle along a streamline is given by the integration of

$$
\frac{d s(t)}{d t}=v[s(t)],
$$

where $v(s)$ is the stream-wise velocity magnitude. The latter is the Lagrangian velocity of a transported particle along its own streamline that can be measured with microscopy and tracking techniques, in units of $[\mathrm{m} / \mathrm{s}]$. In order to project the stream-wise traveled distance along a trajectory $s(t)$ onto linear distance $x(t)$ from the inlet, we use the concept of advective tortuosity $\chi[36]$, a macroscopic quantity which compares average streamline length to linear distance. Tortuosity can be determined by the ratio of the mean absolute velocity and the mean velocity in $x$-direction [43]. Thus, we set $x(t)=s(t) / \chi$, which gives

$$
\frac{d x(t)}{d t}=\frac{v[s(t)]}{\chi} .
$$


In the following, we set $\chi=1$ for simplicity, but in an experimental framework it could be measured in terms of the individual transported colloids trajectories (through microscopy and time lapse imaging) and medium size. Colloids may attach as they move through the medium: we model this process as a first-order kinetics, in analogy to CFT. Here, however, we account for spatial variability of the attachment rate $k=k(x)$ as colloids move through the heterogeneous pore space. This variability is caused by the changing distance between a colloid and the grains surface, as discussed in more detail below. Moreover, we consider that the pore velocity is also a local function of space $v=v(x)[32,35]$ Thus, the distribution $c(x, t)$ of suspended particles corresponding to (6) is given by

$$
\frac{\partial c(x, t)}{\partial t}+\frac{\partial}{\partial x} v(x) c(x, t)=-k(x) c(x, t),
$$

where the right hand side accounts for colloid attachment at the grain surfaces. The steady state profile $c(x)$ is given by:

$$
c(x)=\frac{v(0)}{v(x)} \exp \left[-\int_{0}^{x} d x^{\prime} \frac{k\left(x^{\prime}\right)}{v\left(x^{\prime}\right)}\right],
$$

where the position $x=0$ is the colloids continuous injection point (inlet), as detailed in Appendix A. A. The steady state profile $a(x)$ of the attached colloids is defined by:

$$
a(x)=\int_{0}^{\infty} d t^{\prime} k(x) c\left(x, t^{\prime}\right)
$$

and it requires the transient solution of eq. (7) for $c(x, t)$. In Appendix A, we derive the steady state deposition profile

$$
a(x)=\frac{k(x)}{v(x)} \exp \left[-\int_{0}^{x} d x^{\prime} \frac{k\left(x^{\prime}\right)}{v\left(x^{\prime}\right)}\right] .
$$

Note that this expression differs from equation (3), since it explicitly accounts for the spatial dependence of $v$ and $k$. The fluid velocity in a porous material varies over length scales characteristic of the medium geometry $[29,36]$. Thus, we approximate each trajectory as composed by a number of segments with different lengths $\lambda_{n}$ during which the velocity $v_{n}$ is nearly constant. The length $\lambda_{n}$ is related to the correlation length of stream-wise velocity and of the order of a pore length, typically measured in $[\mathrm{m}][44,45]$. Within a pore, a colloid travels at a nearly constant distance from the grain wall, like in a circular pipe (see $1 \mathrm{c}$-d-e). Therefore, we assume that also the attachment rate $k$, of the transported colloid, remains constant within each pore, but it is variable between pores. This piecewise view of the pore-scale fluid velocity is consistent with previous observations and assumptions that Lagrangian velocities decorrelate on distances of the order of the pore lengths $\lambda[31,32,38,46-48]$. We assume that velocity $v$, attachment rate $k$ and pore size $\lambda$ are independent random variables and distributed according to the probability density functions (PDFs) $p_{v}(v), p_{k}(k)$ and $\psi(\lambda)$ respectively. The macroscopic filtration phenomenon is, thus, the result of these microscopic displacement and attachment mechanisms. Expression (10) denotes the density of attached particles along a single streamline. For a single realization of the stochastic processes $\left\{\lambda_{n}, k_{n}, v_{n}\right\}$ it reads as

$$
a(x)=\frac{k_{n_{x}}}{v_{n_{x}}} \exp \left[-\sum_{i=1}^{n_{x}-1} \frac{k_{i}}{v_{i}} \lambda_{i}-\frac{k_{n_{x}}}{v_{n_{x}}}\left(x-x_{n_{x}}\right)\right],
$$

where after $n$ steps the position is given by

$$
x_{n+1}=x_{n}+\lambda_{n}
$$

and the number $n_{x}$ of steps needed to get as close as possible to $x$ is $n_{x}=\max \left(n \mid x_{n} \leq x\right)$.

\section{B. Ensemble average}

The global profile of attached particles is obtained through the average of $a(x)$ over many realizations of $\{\lambda, k, v\}$,

$$
a(x) \equiv\langle a(x)\rangle=\left\langle\frac{k_{n_{x}}}{v_{n_{x}}} \exp \left[-\sum_{i=1}^{n_{x}-1} \frac{k_{i}}{v_{i}} \lambda_{i}\right] \exp \left[-\frac{k_{n_{x}}}{v_{n_{x}}}\left(x-x_{n_{x}}\right)\right]\right\rangle,
$$


where the angular brackets denote the ensemble average. In the following, we will split the contribution to the deposition profile provided by the first $n_{x}-1$ steps, from the one provided by the last segment of length $x-x_{n_{x}}$. Note that expression (13) depends on the ratio $k_{n} / v_{n}$ rather than $k_{n}$ and $v_{n}$ individually. Thus, we define the new stochastic variable $\rho_{n}=k_{n} / v_{n}$, whose inverse denotes a length, which is the characteristic filtration distance traveled by a colloid with velocity $v_{n}$ before getting retained with an attachment rate $k_{n}$ along its own path. The PDF of this new quantity, $p_{\rho}(\rho)$, can be expressed as

$$
p_{\rho}(\rho)=\int_{0}^{\infty} d v p_{k}(\rho v) p_{v}(v) .
$$

Intuitively, eq. (13), expresses the probability that particles do not attach (in the previous $n-1$ steps) up the distance $x_{n-1}$ times the probability that they get attached during the last step of length $x-x_{n_{x}}$. The first term of eq. (13) is the Laplace transform of $p_{\rho}(\rho)$

$$
\tilde{p}_{\rho}(x)=\int_{0}^{\infty} d \rho \exp (-\rho x) p_{\rho}(\rho)=\mathrm{E}\left[e^{-\rho x}\right],
$$

which denotes the probability that a colloid is not attached at the distance $x$, and consists in the expectation value of $e^{-\rho x}$. The second term is related to

$$
-\frac{d \tilde{p}_{\rho}(x)}{d x}=\int_{0}^{\infty} d \rho \rho \exp (-\rho x) p_{\rho}(\rho)
$$

through multiplication by the probability $\Psi(x)$

$$
\Psi(x)=\int_{x}^{\infty} d \lambda \psi(\lambda)
$$

that the current segment of length $\lambda$ is longer than $x$. Thus, collecting all these terms, the ensemble average in eq. (13) for the deposition profile can be computed explicitly as (see Appendix B for the full derivation),

$$
\bar{a}(x)=-\int_{0}^{x} d x^{\prime} R\left(x^{\prime}\right) \frac{d \tilde{p}_{\rho}\left(x-x^{\prime}\right)}{d x} \Psi\left(x-x^{\prime}\right),
$$

where

$$
R(x)=\delta(x)+\int_{0}^{x} d x^{\prime} R\left(x^{\prime}\right) \tilde{p}_{\rho}\left(x-x^{\prime}\right) \psi\left(x-x^{\prime}\right) .
$$

The quantity $R(x)$ denotes the joint probability that the particle is at position $x$ and it is not attached. Equation (19) represents the mass conservation: indeed, the right hand side denotes the probability that a colloid traveled up to the position $x^{\prime}$ times the probability that next segment length is $x-x^{\prime}$. In order to de-couple the system of eqs. (18), (19) and to obtain a closed solution for the deposition profile $a(x)$, in the following, we will consider the Laplace transform of eqs. (18), (19). In the appendix we provide the derivation to get the following expression for the Laplace transform of the whole deposition profile:

$$
\tilde{a}(u)=\frac{\left\langle\frac{\rho(1-\tilde{\psi}(u+\rho))}{u+\rho}\right\rangle_{\rho}}{1-\langle\tilde{\psi}(u+\rho)\rangle_{\rho}} .
$$

where $u$ denotes the transformed variable of $x$, which has the same units of $\rho=k / v,[m]^{-1}$. In the following we will explicit the deposition profile $a(x)$ for different small scale parameters $\{\lambda, v, k\}$ distribution. 
III. MICROSCOPIC PROCESSES AND THEIR DISTRIBUTIONS

The introduced model is based on a piece-wise view of the pore-scale structure, transport and attachment mechanisms. We assume that a colloid moves through a pore of length $\lambda_{i}$ by the local flow with a constant velocity $v_{i}$ and may attach at rate $k_{i}$, which changes between transitions of length $\lambda_{i+1}$. This length scale can be interpreted as the physical size of an individual pore, but it may comprise also several pores over which the velocity of transported particles persists such as in high velocities channels [32]. In this framework, the physical meaning of $\lambda$ refers to the to medium structures that are larger than individual pores, but consist of several pores that are well connected, from an hydraulic point of view. We consider two main cases, i) a strongly heterogeneous and ii) a weakly heterogeneous medium. The weakly heterogeneous medium is characterized by a narrow disorder distribution as defined below. The strongly heterogeneous medium is characterized by properties distributed across a wide range of pore size and attachment rates.

Porous materials, whose structural properties do not display large spatial variability, have been widely used in order to investigate the link between small scale fluid mechanics and the overall transport phenomena, for instance packed beads $[47,49]$. In these conditions, the pore size distribution is typically narrow, spanning not much more than one order of magnitude about the mean. According to [49, 50], we will consider a log-normal distribution of the pore size $\lambda$

$$
\psi(\lambda) \sim \frac{1}{\lambda} \exp \left(-\frac{\left(\log \lambda-\mu_{\lambda}\right)^{2}}{2 \sigma_{\lambda}^{2}}\right)
$$

where the parameters $\mu_{\lambda}$ and $\sigma_{\lambda}$ define the mean as $e^{\mu_{\lambda}+\sigma_{\lambda}^{2} / 2}$. The values adopted in the simulations for $v_{0}$ are described in the caption of Figure 2: we used $\mu_{\lambda}=2$ and $\sigma_{\lambda}=0.5$ which corresponds to a mean pore size of 8.4 $l_{c}$, the smallest pore size, which is a reasonable value for a weakly heterogeneous medium (a material whose pore size distribution is not homogeneous, peaked about a single value, with variability spanning over about one order of magnitude). For such approximately uniform structures, the flow has been measured within microfluidics replica of a porous material using video-microscopy and particle imaging velocimetry [47] and it has been shown to have a narrow, exponential Eulerian velocity distribution. The proposed modeling approach is based on particle velocities measured spatially along trajectories, see Eq. (11). Thus, the particle velocity is given by the flux-weighted Eulerian velocity distribution [51]

$$
p_{v}(v) \sim v \exp \left(-\frac{v}{v_{0}}\right)
$$

where $v_{0}$ represents the mean fluid velocity. The values adopted in the simulations for $v_{0}$ are described in the caption of Figure 2: they span the range $[10-500] l_{c}$ per second $\left(l_{c}\right.$ is the smallest pore size). This is a resonable range of values considering that a typical porous medium velocity is $v_{0} \sim 10 \mu \mathrm{m} / \mathrm{s}$ (about $1 \mathrm{~m}$ per day) and typical pore sizes range from $1 \mu \mathrm{m}$ up to few $\mathrm{mm}$. In these conditions, we assume that the local attachment rate $k$ is inversely proportional to the grain size, $k \sim 1 / \lambda$, as discussed in more detail below for the heterogeneous case, thus

$$
p_{k}(k) \sim \frac{1}{k} \exp \left(-\frac{\left(-\log k-\mu_{k}\right)^{2}}{2 \sigma_{k}^{2}}\right) .
$$

\section{B. Strong heterogeneity}

In complex and heterogeneous structures, even though pore size distributions are not easy to measure, recent research [45] has shown that the distribution of pore sizes and throats, measured in clay and sandstone samples with 
different high-resolution and cutting-edge technologies (BIB-SEM, X-ray $\mu$-CT and FIB-SEM image-stacks), turn out to be power law distributed over a broad range of length scales. Similarly, using small-angle neutron scattering and fluid-invasion methods it has been shown [44] that also shale samples display a power law distribution of the pore sizes. Thus, we choose, here, a Pareto distribution for the length $\lambda$

$$
\psi(\lambda)=\frac{\gamma}{l_{c}}\left(\frac{\lambda}{l_{c}}\right)^{-1-\gamma},
$$

where $l_{c}$ represents the smallest, detectable, pore size $\left(\lambda>l_{c}\right)$. From now on we re-scale space in terms of $l_{c}$, but for simplicity we will not change the notation of the spatial variable $x$ and $\lambda$ : in this reference $l_{c}=1$. The local fluid velocity modulus through a power-law distributed pore spaces, has been shown in $2 d[23]$ and $3 d$ [31] to follow also a power-law distribution with an exponential cut-off. Thus, we choose a Gamma-distribution for the fluid velocity $v$,

$$
p_{v}(v)=\frac{1}{v_{0} \Gamma(\beta)}\left(\frac{v}{v_{0}}\right)^{\beta-1} \exp \left(-\frac{v}{v_{0}}\right)
$$

where $v_{0}$ denotes the cut-off velocity. Note that typical values for $\beta$ for heterogeneous flows, characterized by broad distributions, are in the range [0,2], [51], in Table I we list all the values adopted for our simulations. To define the statistical distribution of the attachment rate $k$, we assume that the probability for an individual colloid to get attached and retained by a grain wall depends only on the distance at which it is instantaneously located from a solid surface. Note that a pore is not only characterized by its length, but also by the throat size $r$ representing its smallest constriction. We assume that the distance at which a colloid travels from the grain wall is represented by the pore throat: the smaller the pore is, the closer it may be to a solid grain surface and, thus, the larger its attachment rate $k$. It has been found for several materials $[44,45]$ that the distribution of pore throat size $r$ can also be approximated by a Pareto distribution

$$
\psi_{r}(r)=\frac{\delta}{r_{c}}\left(\frac{r}{r_{c}}\right)^{-1-\delta}, \quad r<r_{c}
$$

which is characterized by the exponent $\delta$ and the smallest detectable pore throat size $r_{c}$. Thus, assuming $k \sim 1 / r$, the lower bounded Pareto-type PDF will results in a power-law distribution with a cut-off for high values of $k$, which we model here by the Gamma distribution

$$
p_{k}(k)=\frac{1}{k_{0} \Gamma(\nu)}\left(\frac{k}{k_{0}}\right)^{\nu-1} \exp \left(-\frac{k}{k_{0}}\right)
$$

where the cut-off $k_{0}$ is associated to the smallest throat diameter $r_{c}$. Note that in general, pore length, pore diameter and fluid velocity may be correlated. Here, we assume for simplicity that they are statistically independent. With the above choices for the distribution of velocities $v$ and attachment rates $k$, we obtain for the PDF $p_{\rho}(\rho)$ of $\rho=k / v$ a Beta prime distribution

$$
p_{\rho}(\rho)=\frac{1}{\rho_{0} B(\beta, \nu)}\left(\frac{\rho}{\rho_{0}}\right)^{\nu-1}\left(1+\frac{\rho}{\rho_{0}}\right)^{-\beta-\nu} .
$$

where $B(\beta, \nu)$ is the Beta function and $\rho_{0}=k_{0} / v_{0}$. As we will discuss later, these particular broad-type distributions reflect the medium heterogeneity and are responsible for the emergency of anomalous behavior in the deposition profile $a$. Please note that the physical quantity $\rho=k / v$ has dimension the inverse of a length and the characteristic $1 / \rho_{0}=v_{0} / k_{0}$ represents the distance traveled by a particle moving with velocity $v_{0}$ before being attached while subjected to the attachment rate $k_{0}$. In the following, we will discuss the predictions of our stochastic model, both analytical and by numerical integration of eq. (13), for 11 sets of parameters $\beta, \gamma, \nu, k_{0}$ and $v_{0}$ as detailed in Table I. 
TABLE I. Parameters for the heterogeneous medium simulations. We run 11 simulations varying the cut-off values $v_{0}$ and $k_{0}$ and the characteristic exponents $\beta, \gamma$ and $\nu$ of the distribution for velocity, attachment rate and pore size, respectively. For each simulation, we report the predicted scaling for short $(\beta-1)$ and large distances $(-\nu-\gamma-1)$. Note that since the space has been rescaled with respect to the minimum pore size $l_{c}$, the characteristic velocity $v_{0}$ is given in units of [s $\left.{ }^{-1}\right]$.

\begin{tabular}{c|c|c|c|c|c|c|c|c|c|c|c}
\hline \hline & $\# 1$ & $\# 2$ & $\# 3$ & $\# 4$ & $\# 5$ & $\# 6$ & $\#$ 7 & $\# 8$ & $\# 9$ & $\# 10$ & $\# 11$ \\
\hline$\beta-[-]$ & 0.6 & 0.6 & 0.6 & 0.6 & 0.2 & 0.4 & 0.6 & 0.8 & 0.6 & 0.6 & 0.6 \\
\hline$\gamma-[-]$ & 0.7 & 0.6 & 0.4 & 0.2 & 0.5 & 0.5 & 0.5 & 0.5 & 0.5 & 0.5 & 0.4 \\
\hline$\nu-[-]$ & 0.7 & 0.4 & 0.3 & 0.15 & 0.3 & 0.3 & 0.3 & 0.3 & 0.3 & 0.3 & 0.4 \\
\hline$k_{0}-\left[\mathrm{s}^{-1}\right]$ & $10^{-2}$ & $10^{-1}$ & 1 & 10 & $10^{-4}$ & $10^{-3}$ & $10^{-2}$ & $10^{-1}$ & $10^{-1}$ & $10^{-2}$ & 1 \\
\hline$v_{0}-\left[\mathrm{s}^{-1}\right]$ & 1 & 10 & $10^{2}$ & $10^{3}$ & 1 & 10 & $10^{2}$ & $10^{3}$ & $10^{2}$ & $10^{3}$ & $10^{2}$ \\
\hline$k_{0} / v_{0}-[-]$ & $10^{-2}$ & $10^{-2}$ & $10^{-2}$ & $10^{-2}$ & $10^{-4}$ & $10^{-4}$ & $10^{-4}$ & $10^{-4}$ & $10^{-3}$ & $10^{-5}$ & $10^{-2}$ \\
\hline$\beta-1$ & -0.4 & -0.4 & -0.4 & -0.4 & -0.8 & -0.6 & -0.4 & -0.2 & -0.4 & -0.4 & -0.4 \\
\hline$-\gamma-\nu-1$ & -2.4 & -2.0 & -1.7 & -1.35 & -1.8 & -1.8 & -1.8 & -1.8 & -1.8 & -1.8 & -1.8 \\
\hline \hline
\end{tabular}

\section{SCALING BEHAVIORS}

\section{A. Weak heterogeneity}

As discussed in the previous section, weak medium heterogeneity is characterized by narrow pore size, fluid velocity and attachment rate distributions characterized by finite mean and variance. This is very important to compute the Laplace transforms involved in eq. (20): in fact, the Laplace transform of the function $f(x)$ is the expectation value $\mathcal{L}[f](u)=E\left[e^{-u x}\right]$. At large distances from the inlet $(x \gg 1 /\langle\rho\rangle)$, this expectation value can be computed considering that the transformed variable gets small values $u+\rho \ll 1$, thus, we expand the Laplace transform of the pore size distribution $\tilde{\psi}(u+\rho)$ close to zero, to get $\tilde{\psi}(u+\rho) \sim 1-(u+\rho)$. This implies that eq. (20) can be rewritten as

$$
\tilde{a}(u)=\frac{\langle\rho\rangle_{\rho}}{\langle u+\rho\rangle_{\rho}}
$$

that can be anti-transformed to get the deposition profile

$$
a(x)=\langle\rho\rangle_{\rho} e^{-x\langle\rho\rangle_{\rho}}
$$

that decay exponentially fast, as predicted by the classical framework proposed by CFT. This result is confirmed by the numerical integration of eq. (13), as shown in Fig. 2.

\section{B. Stong heterogeneity}

The defined microscopic process $\rho$ for a heterogeneous medium is characterized by the characteristic length scale $1 / \rho_{0}$, which defines the distinction between the portion of the system that we consider close and far from the inlet location.

A particle that is retained by the host medium at a distance closer than $1 / \rho_{0}=v_{0} / k_{0}$ from the inlet location, got attached before it could transition from its initial velocity $v$. The characteristic survival time for such particles is of the order of $1 / k_{0}$, thus, the location of attachment is $v / k_{0}$ and it depends only on the initial velocity. In other words, particles experience only their own initial velocity for a time $1 / k_{0}$ after which they attach in a way that is independent 


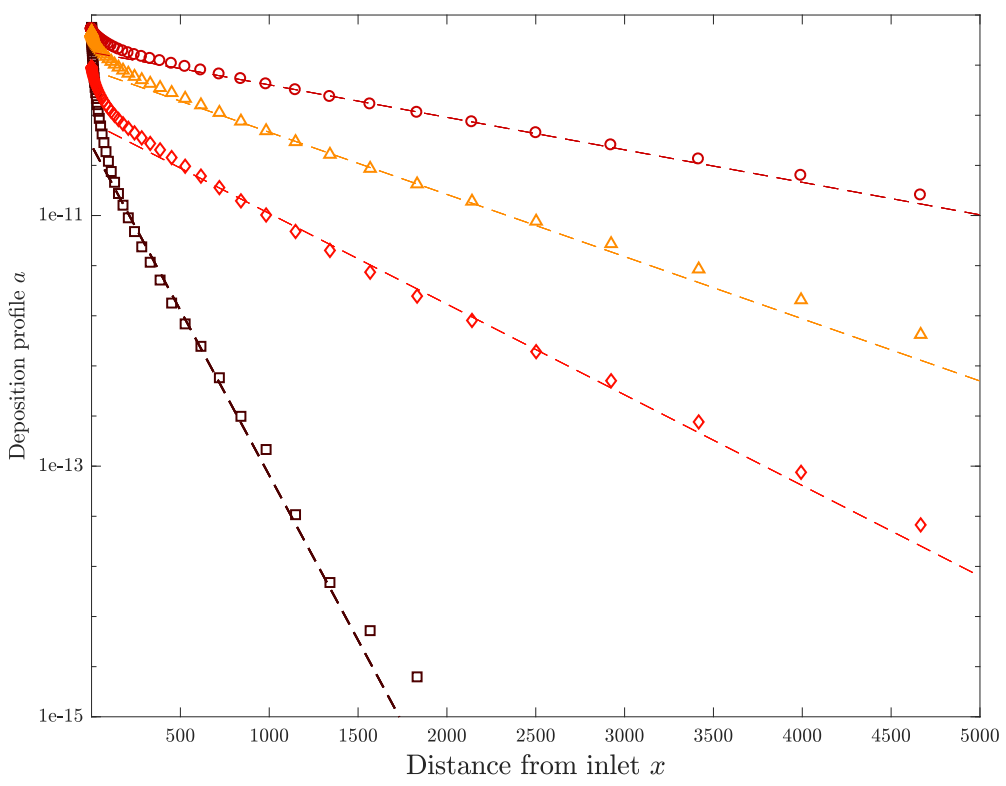

FIG. 2. Deposition profile for a weakly heterogeneous medium. Symbols represent the numerical integration of eq.(3) for the following value of the parameters: $\beta=2, \mu_{\lambda}=2$ and $\sigma_{\lambda}=0.5$ for all sets, set $1(\square), v_{0}=10, \mu_{k}=0.5$ and $\sigma_{k}=0.5$, set $2(\diamond)$, $v_{0}=100, \mu_{k}=0.001$ and $\sigma_{k}=0.5$, set $3(\triangle), v_{0}=200, \mu_{k}=0.5$ and $\sigma_{k}=0.5$ and set $4(\circ) v_{0}=500, \mu_{k}=0.5$ and $\sigma_{k}=0.5$. The dashed lines represent $\exp (-x\langle\rho\rangle)$, where $\langle\rho\rangle$ was computed numerically from the used distributions.

on the specific shape of the $k$ and $\lambda$ distributions. This means that, close to the inlet, the spatial profile of attached particles can be approximated as

$$
a(x) \approx k_{0} p_{v}\left(k_{0} x\right) .
$$

which it is fully determined by the velocity distribution $p_{v}$. The distributions of pore size $\lambda$ and attachment rate $k$ will not play any role since they have not being sampled. The deposition profile scaling for short distances from the inlet location has been computed by the numerical integration of eq. (13) sampling the stochastic variables from the distribution defined in eqs.(25-28),

In Figure 3 (left) is shown the integrated deposition profile for a heterogeneous medium: symbols represent the numerical solution averaged over $2 \cdot 10^{5}$ independent realizations of $k(s), v(s), \lambda(s)$ drawn from distributions eqs. (25-28) and solid lines our model prediction. Using a power law distribution for the pore-scale fluid velocity $p_{v}(v)$, eq. (25), our our model (eq. (31)) results in the power-law scaling

$$
a(x) \sim x^{\beta-1} .
$$

The fitted exponents (see also Appendix D for the fitting procedure) are well matched by our prediction, eq. (32), as shown in Figure3. Different data sets are associated to different choices of the distribution parameters, as described in Table I.

\section{Deposition profiles at large distances from the inlet}

The deposition events that happen after traveling at least $1 / \rho_{0}=v_{0} / k_{0}$ are mainly due to particles that experience low attachment rates and high velocities, so that for each transition $v / k>1$. This imposes bounds for the sampled values of $k$ and $v$. Roughly speaking, the range of low values of $k$ spans the entire power-law regime while only few, 

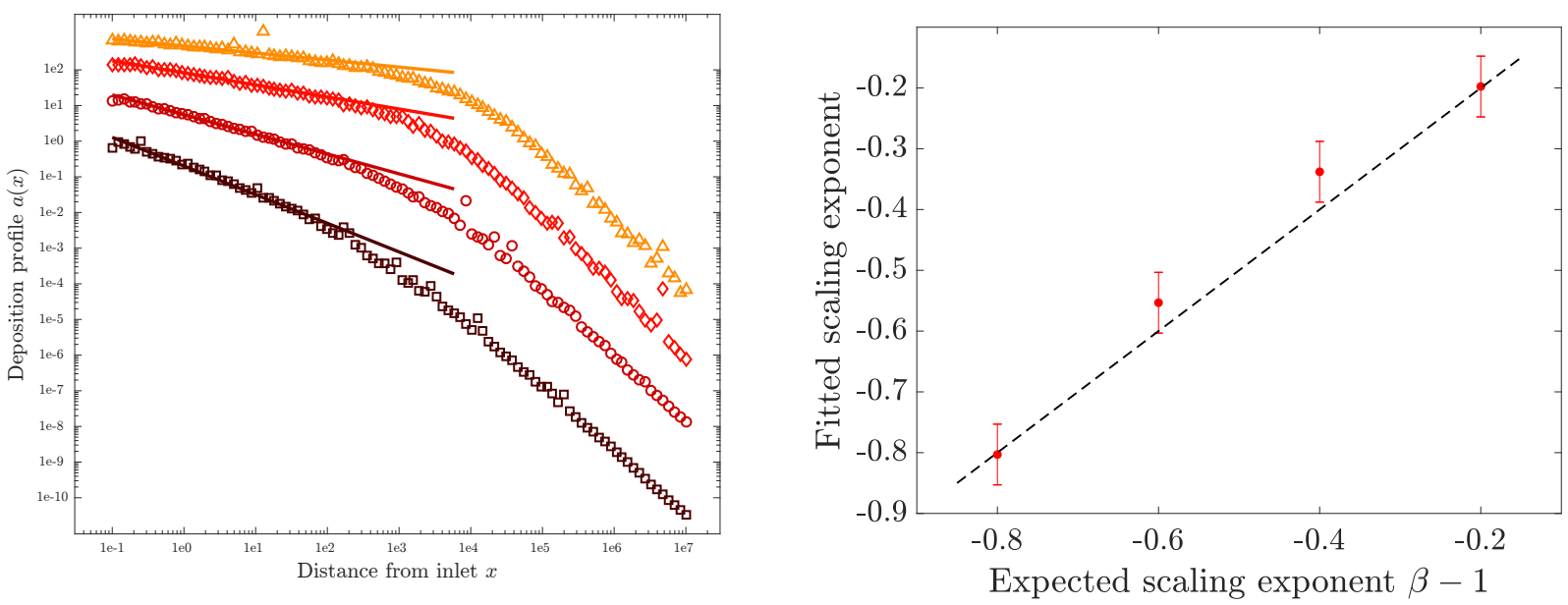

FIG. 3. Left. Deposition profile for heterogeneous medium. Symbols represent the numerical integration of eq. (3) for different values of the parameters that are described in table I: set $5(\square)$, set $6(\circ)$, set $7(\diamond)$ and set $8(\triangle)$. The dashed lines represent a power law fit for the numerical integration of eq.(3), for short distances from inlet. Right. Plot of the fitted values of the exponent $A$, eq.(D1) for the short distance scaling of the deposition profile. The errors are provided by eq.(D2).

high, values of $v>v_{0}$ are sampled to ensure the condition $v / k>1$. Physically, this means that particles sample a broad range of different transition lengths $\lambda$ and attachment rates $k$, at high velocities. Thus we expect that the deposition profile at large distances from the inlet will not depend on the velocity distribution.

As discussed in the appendix, the Laplace transforms of these equations deposition profile $\tilde{a}(u)$ and of the function $\tilde{R}:$

$$
\tilde{a}(u)=\frac{\left\langle\rho(u+\rho)^{\gamma-1}\right\rangle_{\rho}}{\left\langle(u+\rho)^{\gamma}\right\rangle_{\rho}} .
$$

and

$$
\tilde{R}(u)=\frac{1}{\left\langle(u+\rho)^{\gamma}\right\rangle_{\rho}} .
$$

In Appendix $\mathrm{C}$ we show that, considering the defined $p_{\rho}$ and $\psi$ and their expressions in the Laplace space, we find that the profile $a(x)$ of attached particles scales for $x \gg 1 / \rho_{0}$

$$
\bar{a}(x) \propto x^{-\gamma-\nu-1} .
$$

where the exponent $\gamma+\nu+1$ characterizes the particles deposition and reflects the information contained in the pore size and attachment rate distributions. Note that this scaling behavior is valid for any parameter distributions $\psi(\lambda)$ and $p_{k}(k)$ that show the power-law behaviors $\psi(\lambda) \propto \lambda^{-1-\gamma}$ for $\lambda$ smaller than a characteristic length scale $\ell_{c}$ and $p_{k}(k) \propto k^{\nu-1}$ for $k$ smaller than a characteristic rate $k_{0}$, independent from the distribution of velocities. In Figure 4, the symbols represent numerical integration of eq. (13) for different values of the exponents $\gamma, \nu$ and $\beta$, for given $k_{0}$ and $v_{0}$ and the solid lines represent a power law fit on the asymptotic scaling. The fitted exponents are well matched by our prediction, eq. (35), as shown in Figure 4 right.

\section{Regime transitions}

Figure 5 shows the deposition profile associated to the parameters sets 3, 7, 9, and 10 (as described in Table I) for which the exponents $\beta, \gamma$ and $\nu$ are kept constant while the characteristic attachment rate $k_{0}$ and $v_{0}$, that determine 

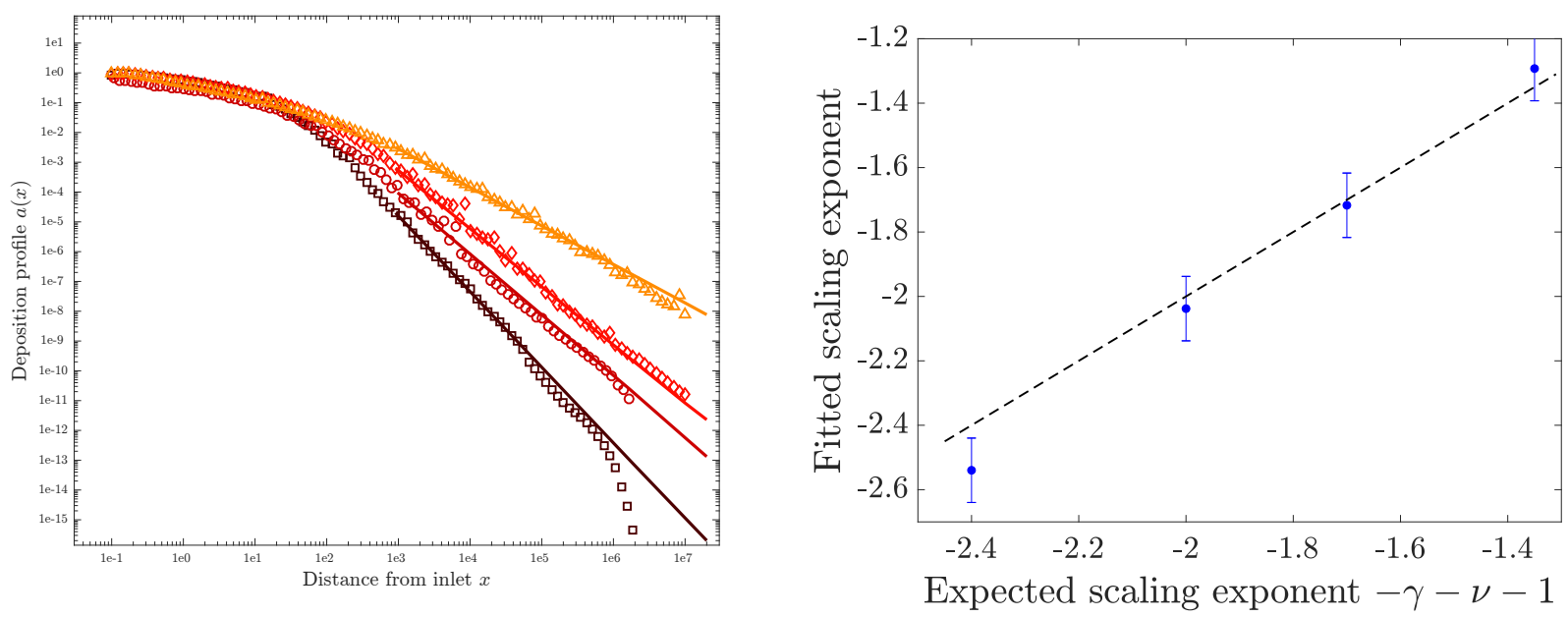

FIG. 4. Left. Deposition profile,symbols represent the numerical integration of eq.(3) for different values of the parameters, as described in table I: set $1(\square)$, set $2(\circ)$, set $3(\diamond)$ and set $4(\triangle)$. The dashed lines represent a power law fit of the numerical integration of eq.(3), for large distances from inlet. Right. Plot of the fitted values of the exponent $A$, eq.(D1) for the large scaling of the deposition profile. The errors are provided by eq.(D2).

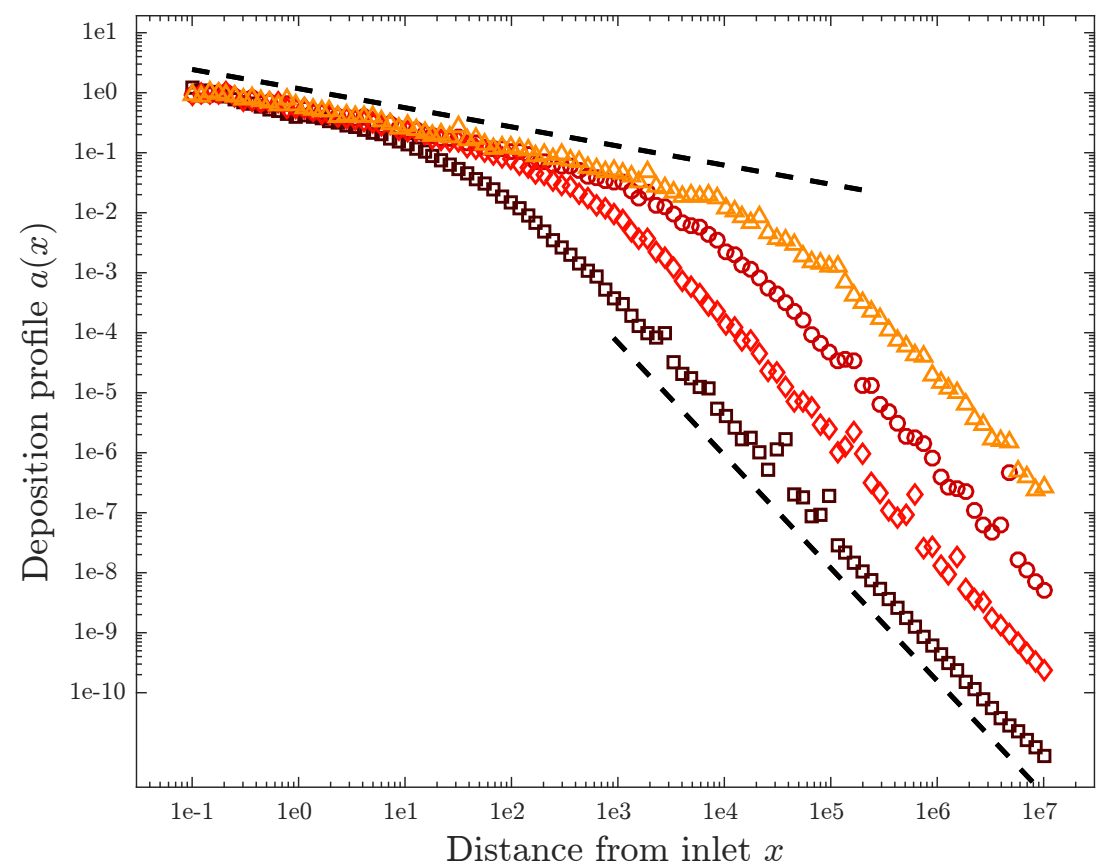

FIG. 5. Deposition profile. Symbols represent the numerical integration of eq. (3) for different values of the parameters, as described in table I: set $11(\square)$, set $7(\circ)$, set $9(\diamond)$ and set $10(\triangle)$. The dashed lines represent the short and large distance scaling predicted by eq. (32) and eq. (35), respectively.

the cut-off for $\rho$, vary as: $10^{-5}, 10^{-4}, 10^{-3}$ and $10^{-2}$. The transition between these two regimes is well predicted by the characteristic filtration scale $1 / \rho_{0}=10^{2}, 10^{3}, 10^{4}$ and $10^{5}$ (note that space has been normalized with respect to the smallest pore size $l_{c}$ ). 


\section{CONCLUSIONS}

We addressed the problem of anomalous deposition profile resulting from the colloids filtration by heterogeneous porous material proposing a novel stochastic model. We predict the Lagrangian steady state deposition profile $a$ as function of traveled distance from the inlet, taking explicitly into account the physical heterogeneity of the host medium structure and velocity distribution in advective dominated regime (neglecting dispersive effects).

In particular, we assumed that each colloid trajectory is composed by a number of segments (the path traveled within each individual pore) of length $\lambda_{i}$ characterized by a velocity $v_{i}$ and attachment rate $k_{i}$ constant. Every time a colloid leaves the pore $i$ to enter the $i+1$ pore of length $\lambda_{i+1}$, it changes its velocity to $v_{i+1}$ and attachment rate to $k_{i+1}$. By averaging the final position of traveling colloids over all possible $\lambda, v$ and $k$, we predict the stationary deposition profile for, weakly heterogeneous and strongly heterogeneous media, as function of the characteristic parameters of the distributions $\psi, p_{v}$ and $p_{k}$, whose definition is consistent with many microscopic observations on real media. The numerical integration of the stationary deposition profile eq.(10), that takes into account the random variability of the three processes $\lambda, v$ and $k$. Our model recovers the classical results of the CFT for weakly heterogeneous media (exponential deposition profile) and for the strongly heterogeneous ones it predicts a power law profile that it is well fitted by our analytical solution for short, eq. (32), and large distances, eq. (35), from the inlet.

The proposed model of filtration, though simple, is the first one that, to the best of our knowledge, brings together the fundamental processes of velocity and correlation length (pore size) to couple them with a first order kinetics. This model can be easily extended to incorporate more realistic, and complex, attachment kinetics including transient attachment-detachment process. In particular, including diffusion and reversible attachment (e.g. with the introduction of a detachment rate or the classical Langmuirian adsorption dynamics) within the main ADE equation, the equivalent Lagrangian formulation can be formulated and numerically integrated within the same solution strategy proposed in our manuscript. Moreover, hgher order kinetic rates, leading to non-linear terms in the ADE formulation, can be analytically treated in terms of perturbative techniques only for restricted range of parameter value for which the whole system can be reduced to a set of linear equations whose solution can be found following the proposed strategy [52].

Finally, we emphasize that we focused our study to the emergency of anomalous steady state deposition profile, providing, an analytical solution for the scaling behavior in the two observed regimes for a strongly heterogeneous medium. Moreover the proposed model can be extended by considering conditional pdf once the inner relations between local velocity field, colloid-surface adhesion force and relative distance are known. This can be addressed by performing single colloid-grain and colloid-grains cluster attachment observations with microscopy techniques. In this case, the size of the particle will also be relevant. Furthermore, other possible extensions include complex time-dependent dynamics emerging from the breakdown of the clean bed assumption (e.g. changing permeability and clogging effect). In this case, aging Continuous Time Random Walk (CTRW) model can be implemented.

Appendix A: Steady state profile

We calculate the steady state profiles of the distributions of the suspended and attached colloids. Thus, we write equation (7) as

$$
\frac{\partial c(x, t)}{\partial t}+v(x) \frac{\partial c(x, t)}{\partial x}=-\left[k(x)+\frac{d v(x)}{d x}\right] c(x, t)
$$

We consider the initial condition $c(x, t=0)=\delta(x)$. The equation for the steady state distribution $c(x)$ is given by

$$
\frac{\partial c(x)}{\partial x}=-\left[\frac{k(x)}{v(x)}+\frac{d \ln v(x)}{d x}\right] c(x) .
$$


It can be solved by separation of variables, which gives:

$$
c(x)=\frac{v(0)}{v(x)} \exp \left[-\int_{0}^{x} \frac{d x^{\prime}}{v\left(x^{\prime}\right)} k\left(x^{\prime}\right)\right] .
$$

The concentration $a(x, t)$ of the attached particles is obtained by mass conservation from

$$
\frac{\partial a(x, t)}{\partial t}=k(x) c(x, t),
$$

whose temporal integration gives eq. (9), by setting the initial condition $a(x, t=0)=0$ : this means that initially there are no attached particles. We focus here on the steady state profile of attached particles, which is given by (9). Thus, we need to solve the transient equation (A1) for $c(x, t)$. To do so, we use the method of characteristics. The characteristic equation for $(\mathrm{A} 1)$ is

$$
\frac{d x\left(t, x_{0}\right)}{d t}=v\left[x\left(t ; x_{0}\right)\right]
$$

where $x\left(t=0, x_{0}\right)=x_{0}$ is the initial value. We define now $g\left(x_{0}, t\right)=c\left[x\left(t ; x_{0}\right), t\right]$ which obeys the following equation

$$
\frac{\partial g\left(x_{0}, t\right)}{\partial t}=-\kappa\left[x\left(t, x_{0}\right)\right] g\left(x_{0}, t\right),
$$

where we defined

$$
\kappa(x)=k(x)+\frac{d v(x)}{d x}
$$

The initial condition is $g\left(x_{0}, t=0\right)=\delta\left(x_{0}\right)$ Separation of variables gives for $g\left(x_{0}, t\right)$

$$
g\left(x_{0}, t\right)=\delta\left(x_{0}\right) \exp \left[-\int_{0}^{t} d t^{\prime} \kappa\left[x\left(t^{\prime}, x_{0}\right)\right] .\right.
$$

We perform now the variable change $t \rightarrow x\left(t ; x_{0}\right)$ in the integral in the exponent, which gives

$$
g\left(x_{0}, t\right)=\delta\left(x_{0}\right) \exp \left[-\int_{x_{0}}^{x\left(t ; x_{0}\right)} \frac{d x^{\prime}}{v\left(x^{\prime}\right)} \kappa\left(x^{\prime}\right)\right]
$$

Note that we can set the lower integration limit in the exponent to 0 because of the Dirac delta. We note that $x_{0}=x_{0}(t, x)$ is a function of $x$ and $t$, thus, we transform back and we obtain for $c(x, t)$,

$$
c(x, t)=\delta\left(x_{0}\right) \exp \left(-\int_{0}^{x} d x^{\prime}\left[\frac{k\left(x^{\prime}\right)}{v\left(x^{\prime}\right)}+\frac{d \ln v\left(x^{\prime}\right)}{d x^{\prime}}\right]\right),
$$

This expression can be further simplified to

$$
c(x, t)=\delta\left(x_{0}\right) \frac{v(0)}{v(x)} \exp \left[-\int_{0}^{x} d x^{\prime} \frac{k\left(x^{\prime}\right)}{v\left(x^{\prime}\right)}\right],
$$

where we used definition (A7). We obtain now the steady state distribution of the attached colloids by inserting (A11) into (9), which gives

$$
a(x)=k(x) \int_{0}^{\infty} d t^{\prime} \delta\left[x_{0}\left(t^{\prime}, x\right)\right] \exp \left[-\int_{0}^{x} \frac{d x^{\prime}}{v\left(x^{\prime}\right)} k\left(x^{\prime}\right)\right] .
$$


In order to simplify this expression, we note that

$$
\delta\left[x_{0}(t, x)\right]=\frac{\delta\left(t-t_{0}\right)}{\left|\frac{d x_{0}(t, x)}{d t}\right|}=\frac{\delta\left(t-t_{0}\right)}{v\left[x_{0}(t ; x)\right]}=\frac{\delta\left(t-t_{0}\right)}{v(0)},
$$

since $t_{0}$ is the solution of $x_{0}(t ; x)=x$. Thus, we obtain

$$
a(x)=\frac{k(x)}{v(x)} \exp \left[-\int_{0}^{x} \frac{d x^{\prime}}{v\left(x^{\prime}\right)} k\left(x^{\prime}\right)\right] .
$$

\section{Appendix B: Stochastic model}

To compute the expectation value in eq. (13), we need to remove the dependence on the index $n_{x}$, representing the number of steps required to get as close as possible to the location $x$ without exceeding it, that is different for each trajectory. Therefore, we make use of the Kronecker delta $\delta_{n, n_{x}}$

$$
a(x)=\sum_{n=1}^{\infty}\left\langle\frac{k_{n}}{v_{n}} \exp \left[-\sum_{i=1}^{n-1} \frac{k_{i}}{v_{i}} \lambda_{i}-\frac{k_{n}}{v_{n}}\left(x-x_{n}\right)\right] \delta_{n, n_{x}}\right\rangle,
$$

so that $\delta_{n, n_{x}}$ partitions the probability space into disjoint sets and the expectation value in (13) became the sum of expectation values. The last path of size $x-x_{n}$ traveled by a colloid before getting attached, must be smaller or equal to the length of the last segment $\lambda_{n}$. Then, we express the Kronecker delta in terms of the Indicator Function $\mathcal{I}(\cdot)$ that is equal to 1 if the argument is true, 0 otherwise:

$$
a(x)=\sum_{n=1}^{\infty}\left\langle\frac{k_{n}}{v_{n}} \exp \left[-\sum_{i=1}^{n-1} \frac{k_{i}}{v_{i}} \lambda_{i}-\frac{k_{n}}{v_{n}}\left(x-x_{n}\right)\right] \mathcal{I}\left(0<x-x_{n}<\lambda_{n}\right)\right\rangle
$$

Finally, we introduce the Dirac delta function $\delta\left(x_{n}-x^{\prime}\right)$ and we integrate overall possible values for $x^{\prime}$ to eliminate the dependence on $x_{n}$ which is different for each considered colloid. Thus,

$$
a(x)=\sum_{n=1}^{\infty} \int_{0}^{x} d x^{\prime} \delta\left(x_{n}-x^{\prime}\right)\left\langle\frac{k_{n}}{v_{n}} \exp \left[-\sum_{i=1}^{n-1} \frac{k_{i}}{v_{i}} \lambda_{i}-\frac{k_{n}}{v_{n}}\left(x-x^{\prime}\right)\right] \mathcal{I}\left(0<x-x^{\prime}<\lambda_{n}\right)\right\rangle .
$$

Since the random variables $k$ and $v$ always appear coupled as $k / v$, we simplify the notation by the introduction of a new stochastic variable $\rho=k / v$, as discussed in the main manuscript. Please note that, since the stochastic processes at each step are independent, the term that depends only on the index $n$ can be re-written without the $n$-dependence, as $\lambda_{n}=\lambda$ and $\rho_{n}=\rho$.

$$
a(x)=\sum_{n=1}^{\infty} \int_{0}^{x} d x^{\prime} \delta\left(x_{n}-x^{\prime}\right)\left\langle\exp \left[-\sum_{i=1}^{n-1} \rho_{i} \lambda_{i}\right]\right\rangle\left\langle\rho e^{-\rho\left(x-x^{\prime}\right)} \mathcal{I}\left(0<x-x^{\prime}<\lambda\right)\right\rangle .
$$

Following [53] we, now, exchange the integration over $x^{\prime}$ with summation over $n$ since the two variables are independent and we break the expectation value in two terms, one averaging over all indices up to $n-1$ and the one averaging over the last index $n$ :

$$
a(x)=\int_{0}^{x} d x^{\prime} \sum_{n=1}^{\infty} R_{n}\left(x^{\prime}\right)\left\langle\rho e^{-\rho\left(x-x^{\prime}\right)} \mathbb{I}\left(0 \leq x-x^{\prime} \leq \lambda\right)\right\rangle
$$

where we have defined

$$
R_{n}(x)=\left\langle\delta\left(x-x^{\prime}\right) \exp \left[-\sum_{i=1}^{n-1} \rho_{i} \lambda_{i}\right]\right\rangle
$$


which represents the probability for a colloid of not being attached after $n-1$ steps and getting to the distance $x_{n}$ from the injection point. To derive an expression for $R$ that does not depend on the index $n$, we consider that $x_{n+1}=x_{n}+\lambda_{n}$

$$
R_{n+1}(x)=\left\langle\delta\left(x-x_{n}-\lambda_{n}\right) \exp \left[-\sum_{i=1}^{n} \rho_{i} \lambda_{i}\right] \exp [-\rho \lambda]\right\rangle .
$$

Moving the variable $x_{n}$ into $x^{\prime}$, by the introduction of $\delta\left(x_{n}-x^{\prime}\right)$ we get:

$$
R_{n+1}(x)=\int d x^{\prime}\left\langle\delta\left(x_{n}-x^{\prime}\right) \exp \left[-\sum_{i=1}^{n-1} \rho_{i} \lambda_{i}\right]\right\rangle\left\langle\delta\left(x-x^{\prime}-\lambda\right) \exp [-\rho \lambda]\right\rangle
$$

Please note that the two expectation values that appear in the previous expression have to be computed over all possible values for the two independent stochastic variables $\rho$ and $\lambda$, in other words $\langle\cdot\rangle=\langle\cdot\rangle_{\rho, \lambda}$. Therefore, we can explicitly calculate the average over the random jumps $\lambda$, as

$$
\begin{array}{r}
\left\langle\delta\left(x-x^{\prime}-\lambda\right) \exp [-\rho \lambda]\right\rangle_{\rho, \lambda}=\left\langle\int d \lambda \psi(\lambda) \delta\left(x-x^{\prime}-\lambda\right) \exp [-\rho \lambda]\right\rangle_{\rho} \\
=\left\langle\psi\left(x-x^{\prime}\right) e^{-\rho\left(x-x^{\prime}\right)}\right\rangle_{\rho}
\end{array}
$$

where $\langle\cdot\rangle_{\rho}$ denotes the expectation value over the stochastic variable $\rho$ alone, from which we obtain:

$$
R_{n+1}=\int_{-\infty}^{\infty} d x^{\prime} R_{n}\left(x^{\prime}\right)\left\langle e^{-\rho\left(x-x^{\prime}\right)} \psi\left(x-x^{\prime}\right)\right\rangle_{\rho} .
$$

We can now define

$$
R(x)=\sum_{n=1}^{\infty} R_{n}(x)=R_{1}+\sum_{n=1}^{\infty} R_{n+1}(x)
$$

and we note that $R_{1}=\delta(x)$, so that from the previous expression we can derive (19).

\section{Appendix C: Scaling of the spatial profile of attached colloids}

To compute the dependence of the deposition profile $a(x)$ from the distribution of the stochastic processes $\rho$ and $\lambda$, we compute the Laplace transform eq. (19) $\tilde{a}(u)=\mathcal{L}\{a\}(u)$, where $u$ denotes the transformed variable $x \rightarrow u$. Noting that $a(x)$ in eq.(18) is expressed as a convolution product, its spatial Laplace transform (with respect to the variable $x)$ is the product between the Laplace transforms of the two functions:

$$
\tilde{a}(u)=\mathcal{L}\{R\}(u)=\mathcal{L}\left\{\left\langle\rho e^{-\rho x}\left(1-\int_{0}^{x} d \lambda \psi(\lambda)\right)\right\rangle_{\rho}\right\} .
$$

We now consider the Laplace transform properties i) $\mathcal{L}\{\delta\}(u)=1$ and ii) $\mathcal{L}\left\{e^{\rho x} f(x)\right\}=F(\rho+u)$, where $F=\mathcal{L}\{f\}$ is the Laplace transform of the function $f(x)$. Therefore, the Laplace transform of $R$ is

$$
\mathcal{L}\{R\}(u)=\frac{1}{1-\mathcal{L}\{\psi\}(\rho+u)}
$$

where $\tilde{\psi}(u+\rho)=\mathcal{L}\{\psi\}(\rho+u)$ is the Laplace transform of the pore size distribution. Please note that the transformed variable $u$ has units as $\left[x^{-1}\right]$ which is the inverse of a length, like $\rho=k / v$. Considering the Laplace transform of the second term in eq. (C1), we get

$$
\mathcal{L}\left\{\left\langle\rho e^{-\rho x}\left(1-\int_{0}^{s} d \lambda \psi(\lambda)\right)\right\rangle_{\rho}\right\}=\left\langle\frac{\rho(1-\tilde{\psi}(u+\rho))}{u+\rho}\right\rangle_{\rho}
$$


where, due to the independence of $\lambda$ and $\rho$, we exchanged the order of computation for the expectation value and Laplace transformation, then, we exploit the Laplace transform property

$$
\mathcal{L}\left\{\int_{0}^{x} d x^{\prime} f\left(x^{\prime}\right)\right\}=\frac{F(u)}{u} .
$$

Finally, substituting eq.(C2) and eq.(C4) into eq.(C1) we get the following expression for the Laplace transform of the whole deposition profile:

$$
\tilde{a}(u)=\frac{\left\langle\frac{\rho(1-\tilde{\psi}(u+\rho))}{u+\rho}\right\rangle_{\rho}}{1-\langle\tilde{\psi}(u+\rho)\rangle_{\rho}} .
$$

\section{Heterogeneous medium}

The inverse transform of the previous expression $a(x)=\mathcal{L}^{-1}\{\tilde{a}(u)\}$ is the deposition profile we are looking for. Please note that that the overall deposition profile directly depends on the distributions of the stochastic processes governing the micro-scale attachment and transport mechanisms. Thus, to obtain an explicit expression for the deposited particle eq. (C5) in this regime, we have to calculate the Laplace transform of the pore size distribution $\psi(\lambda)$. It is possible to show [54] that the Laplace transform of the power law defined in eq. (24) can be expressed as the series $\tilde{\psi}(u) \approx 1-\left(l_{c} u\right)^{\gamma}+o\left(\left(l_{c} u\right)^{\gamma}\right)$. Substituting this expression into (C5) we find:

$$
\tilde{a}(u)=\frac{\left\langle\rho(u+\rho)^{\gamma-1}\right\rangle_{\rho}}{\left\langle(u+\rho)^{\gamma}\right\rangle_{\rho}} .
$$

To evaluate the Laplace inverse transform of the previous expression, it has been shown [53] (in the framework of a reactive-diffusive system) that the behavior of eq.(C6) is dominated by the numerator alone. Therefore, to compute the expectation value over the stochastic variable $\rho$ we make use of eq.(28) and we obtain

$$
\left\langle\rho(u+\rho)^{\gamma-1}\right\rangle_{\rho}=\int d \rho \rho^{\nu}\left(\rho_{0}+\rho\right)^{-\beta-\nu}(u+\rho)^{\gamma-1} .
$$

Since we are interested in the particles deposition at distances larger than the characteristic length $1 / \rho_{0}$, we consider eq. (C7) in the limit $\rho \ll \rho_{0}$, obtaining:

$$
\left\langle\rho(u+\rho)^{\gamma-1}\right\rangle_{\rho} \sim u^{\gamma+\nu} .
$$

To compute the inverse Laplace transform of the previous expression, we exploit the two properties of the Laplace transform

$$
\mathcal{L}^{-1}\left\{u^{-a} \Gamma(a)\right\}=x^{a-1}, \text { for } a>0
$$

and

$$
\mathcal{L}^{-1}\left\{\tilde{f}^{(k)}(u)\right\}=(-1)^{k} x^{k} f(s),
$$

where $\tilde{f}^{(k)}$ is the $k$-th derivative and we, finally, get eq. (35).

\section{Appendix D: Data fitting procedure}

To fit a power law $f(x)=A+x^{B}$ we look for the values of $A$ and $B$ that minimize the distance between the model, $f(x)$, and the data set. To do that, we consider $g(x)=\log [f(x)]=\log (A)+B \log (x)$ and we minimize this function 
performing a linear regression procedure with respect to the variable $y=\log (x)$. Minimizing the squared discrepancy between a data set and a linear model, we get [55]:

$$
A=\frac{\sum_{i} X_{i}^{2} \sum_{k} Y_{k}-\sum_{i} X_{i} \sum_{i} X_{i} Y_{i}}{N \sum_{i} X_{i}^{2}-\left(\sum_{i} X_{i}\right)^{2}}, \quad B=\frac{N \sum_{i} X_{i} Y_{i}-\sum_{i} X_{i} \sum_{k} Y_{k}}{N \sum_{i} X_{i}^{2}-\left(\sum_{i} X_{i}\right)^{2}}
$$

where $X_{i}=\log \left(x_{i}\right)$ and $Y_{i}=\log \left(g_{i}\right)$. The error on the estimation of the fitted parameter $B$, representing the characteristic power-law scaling, is computed following [55]:

$$
\delta B=\sigma_{y} \sqrt{\frac{\sum x_{i}^{2}}{N \sum_{i} x_{i}^{2}-\left(\sum_{i} x_{i}\right)^{2}}}, \quad \sigma_{y}=\sqrt{\frac{1}{N-2} \sum_{i=1}^{N}\left(y_{i}-A-B x_{i}\right)^{2}} .
$$

[1] J. N. Israelachvili, Intermolecular and Surface Forces (Elsevier, Academic Press, 2011).

[2] S. D. Keesstra, V. Geissen, K. Mosse, S. Piiranen, E. Scudiero, M. Leistra, and L. van Schaik, "Soil as a filter for groundwater quality," Current Opinion in Environmental Sustainability 4 (5), 507-516 (2012).

[3] Karwan Diana L. and Saiers James E., "Hyporheic exchange and streambed filtration of suspended particles," Wsater Resour. Res. 48 (1), 2011 WR011173 (2012).

[4] Jaffrin Michel Y., "Dynamic shear-enhanced membrane filtration: A review of rotating disks, rotating membranes and vibrating systems," Journal of Membrane Science 324, 7-25 (2008).

[5] Pillai Sumitra A., Chobisa Dhawal, Urimi Dileep, and Ravind Nagasuri, "Filters and filtration: A review of mechanisms that impact cost, product quality and patient safety," J. Pharm. Sci. Res. 8 (5), 271-278 (2016).

[6] W. P. Johnson, "Why variant colloid transport behaviors emerge among identical individuals in porous media when colloid-surface repulsion exists," Environ. Sci. Technol. 10.1021/acs.est.8b00811 (2018).

[7] J. Bear, Dynamics of Fluids in Porous Media (Elsevier, New York, 1972).

[8] M. J. Blunt et al., "Pore-scale imaging and modelling," Adv. Water Resour. 51, 97-216 (2013).

[9] R. A. Freeze and J. A. Cherry, Groundwater (Prentice Hall, 1979).

[10] A. Boisson, P. de Anna, O. Bour, T. Le Borgne, T. Labasque, and L. Aquilina, "Reaction chain modeling of denitrification reactions during a push-pull test," J. Contaminant Hydrol. 148, 1-11 (2013).

[11] L. Cueto-Felgueroso and R. Juanes, "Nonlocal interface dynamics and pattern formation in gravity-driven unsaturated flow through porous media," Phys. Rev. Lett. 101, 244504 (2008).

[12] M. L. Szulczewski, C. W. MacMinn, H. J. Herzog, and R. Juanes, "Lifetime of carbon capture and storage as a climatechange mitigation technology," Proc. Natl. Acad. Sci. 109, 5185 (2012).

[13] D. Wu, H. Liu, M. Xie, H. Liu, and W. Sun, "Experimental investigation on low velocity filtration combustion in porous packed bed using gaseous and liquid fuels," Exp. Ther. Fluid Sci. 36, 169-177 (2012).

[14] M. E. Nelson and T. R. Ginn, "Colloid filtration theory and the happel sphere-in-cell model revisited with direct numerical simulation of colloids," Langmuir 21, 2173-2184 (2005).

[15] J. Happel, "Viscous flow in multiparticle system: Slow motion of fluids relative to beds of sperical particles," Environ. Sci. Technol. 37, 616-623 (2003).

[16] K. M. Yao, M. T. Habibian, and C. O. O'Melia, "Water and waste filtration. concepts and applications," Environ. Sci. Technol. 5, 1105-1112 (1971).

[17] Rajagopalan Rajamani and Tien Chi, "Trajectory analysis of deep-bed filtration with the sphere-in-cell porous media model," AIChE Journal 22,No 3, 523-533 (1976).

[18] N. Tufenkji, "Modeling microbial transport in porous media: Traditional approaches and recent developments," A.I.Cg.E. Journal 4, 197-201 (1958).

[19] N. Tufkenji, J. A. Redman, and M. Elimelech, "Interpeting deposition patterns of microbial particles in laboratory-scale column experiments," Environ. Sci. Technol. 37, 616-623 (2003).

[20] A. Cortis, T. Harter, L. Hou, E. R. Atwill, and A. I. Packman, "Transport of cryptosporidium parvum in porous media: Long-term elution experiments and continuous random walk filtration modeling," Water Resour. Res. 42, W12S13 (2006).

[21] A. Adin and R. Rajagopalan, "Breakthrough curves in granual media filtration," J. Environ. Eng. 115, 785-798 (1989).

[22] Leung Wallace Woon-Fong, Hung Chi-Ho, and Yuen Ping-Tang, "Experimental investigation on continuous filtration of sub-micron aerosol by filter composed of dual-layers including a nanofiber layer," Aerosol Sci Technol. 43, 1174-1183 (2009). 
[23] P. de Anna, B. Quaife, G. Biros, and R. Juanes, "Prediction of velocity distribution from pore structure in simple porous media," Phys. Rev. Fluids 2, 124103 (2017).

[24] N. Toride and S. A. Bradford, "A stochastic model for colloid transport and deposition," J. Environ. Qual. 36, 1346-1356 (2007).

[25] S. A. Bradford, J. Simunek, M. Bettahar, M. T. van Genuchten, and S. R. Yates, "Modeling colloid attachment, straining and exclusion in saturated porous media," Environ. Sci. Technol. 37(10), 2242-2250 (2003).

[26] M. Elimelech, Xi Jia, J. Gregory, and R. A. Williams, Particle Deposition and Aggregation - Measurement, Modeling and Simulation (Butterworth Heinemann, 1995).

[27] P. De Anna, J. Jimenez-Martinez, H. Tabuteau, R. Turuban, T. Le Borgne, M. Derrien, and Y. Méheust, "Mixing and reaction kinetics in poroous media: an experimental pore scale quantification," Environ. Sci. Technol. 48, 508-516 (2014).

[28] A. M. Tartakovsky, D. M. Tartakovsky, and P. Meakin, "Stochastic Langevin model for flow and transport in porous media," Phys. Rev. Lett. 101, 044502 (2008).

[29] Veronica L Morales, Marco Dentz, Matthias Willmann, and Markus Holzner, "Stochastic dynamics of intermittent porescale particle motion in three-dimensional porous media: Experiments and theory," Geophysical Research Letters 44, 9361-9371 (2017).

[30] I. Battiato, D. M. Tartakovsky, A. M. Tartakovsky, and T. Scheibe, "On breakdown of macroscopic models of mixingcontrolled heterogeneous reactions in poroous media," Adv. Water Resour. 32, 1664-1673 (2009).

[31] M Dentz, M. Icardi, and J. J. Hidalgo, "Mechanism of dispersion in a porous medium," J. Fluid Mech. 841, 851-882 (2018).

[32] P. de Anna, T. Le Borgne, M. Dentz, A. M. Tartakovsky, D. Bolster, and P. Davy, "Flow intermittency, dispersion, and correlated continuous time random walks in porous media," Phys. Rev. Lett. 110, 184502 (2013).

[33] M. J. Blunt, "Flow in porous media-pore network models and multiphase flow," Curr. Opin. Colloid Interface Sci. 6, 197-207 (2001).

[34] B. Bijeljic, P. Mostaghimi, and M. J. Blunt, "Signature of non-Fickian solute transport in complex heterogeneous porous media," Phys. Rev. Lett. 107, 204502 (2011).

[35] M. Holzner, V. L. Morales, M. Willmann, and M. Dentz, "Intermittent lagrangian velocities and accelerations in threedimensional porous medium flow," Phys. Rev. E 92, 013015 (2015).

[36] A. Puyguiraud, P. Gouze, and M. Dentz, "Stochastic dynamics of lagrangian pore-scale velocities in three-dimensional porous media," Water Resour. Res. under review (2018).

[37] DR Lester, Guy Metcalfe, and MG Trefry, "Is chaotic advection inherent to porous media flow?" Phys. Rev. Lett. 111, 174101 (2013).

[38] P.K. Kang, P. de Anna, J.P. Nunes, B. Bijeljic, M. J. Blunt, and R. Juanes, "Pore-scale intermittent velocity structure underpinning anomalous transport through 3-d porous media," Geophys. Res. Lett. GL061475 (2014).

[39] Vivek Kapoor, Chad T Jafvert, and Dennis A Lyn, "Experimental study of a bimolecular reaction in Poiseuille flow," Water resources research 34, 1997-2004 (1998).

[40] Carolyn M. Gramling, Charles F. Harvey, and Lucy C. Meigs, "Reactive transport in porous media: a comparison of model prediction with laboratory visualization," Environmental Science \& Technology 36, 2508-2514 (2002).

[41] T. Le Borgne, M. Dentz, P. Davy, D. Bolster, J. Carrera, J.R. de Dreuzy, and O. Bour, "Persistence of incomplete mixing: A key to anomalous transport," Phys. Rev. E 84, 015301 (2011).

[42] P. de Anna, M. Dentz, A.M. Tartakovsky, and T. Le Borgne, "The filamentary structure of mixing fronts and its control on reaction kinetics in porous media flows," Geophys. Res. Lett. 41, 4586-4593 (2014).

[43] A Koponen, M Kataja, and Jv Timonen, "Tortuous flow in porous media," Physical Review E 54, 406 (1996).

[44] J. Zhao, Z. Jin, Q. Hu, Z. Jin, T. J. Barber, Y. Zhang, and M. Bleuel, "Integrating sans and fluid invasion methods to characterize pore structure of typical american shale oil reservoirs," Sci. Rep. 7:15413 (2017).

[45] Desbois G. Hemes, S., J. L. Urai, B. Schroppel, and J. O. Schwarz, "Multi-scale characterization of porosity in boom clay (hades-level, mol, belgium) using a combination of x-ray $\mu$-ct, 2d bib-sem and fib-sem tomography," Microporous Mesoporous Mater. 2018, 1-20 (2015).

[46] PG Saffman, "A theory of dispersion in a porous medium," Journal of Fluid Mechanics 6, 321-349 (1959).

[47] S. S. Datt, H. Chiang, T. S. Ramakrishnan, and D. A. Weitz, "Spatial fluctuations of fluid velocities in flow through a three-dimensional porous medium," Physical Review Letters 111 (2013).

[48] Marco Dentz, Peter K Kang, Alessandro Comolli, Tanguy Le Borgne, and Daniel R Lester, "Continuous time random walks for the evolution of lagrangian velocities," Physical Review Fluids 1, 074004 (2016). 
[49] I. Gueven, S. Frijters, H. Harting, S. Luding, and H. Steeb, "Hydraulic properties of porous sintered glass bead systems," Granular Matter , 19:28 (2017).

[50] Kazutaka Mizutani, Hideki Minagawa, Kotaro Ohga, Naoya Takahara, Yasuhide Sakamoto, Takeshi Komai, Tsutomu Yamaguchi, and Hideo Narita, "Relation between pore size distribution and permeability of sediment consist of glass beads and sand," Journal of the Japanese Association for Petroleum Technology 75, 164-176 (2010).

[51] C. Alessandro and M. Dentz, "Anomalous dispersion in correllated porous media: a coupled continuous time random walk approach," Eur. Phys. J. B. 90, 166-184 (2017).

[52] Y. Kuramoto, Chemical Oscillations, Waves, and Turbulence (Springer Science \& Business Media, 2012).

[53] G. J. Lapeyre and M. Dentz, "Reaction-diffusion with stochastic decay rates," Phys. Chem. Chem. Phys. 19, 18863-18879 (2017).

[54] J. Klafter and I. M. Solokov, First Steps in Random Walks (Oxford University Press, 2011).

[55] J. R. Taylor, An Introduction to Error Analysis The Study of Uncertainties in Physical Measurements, 2nd ed. (University of Science Books, 1997). 